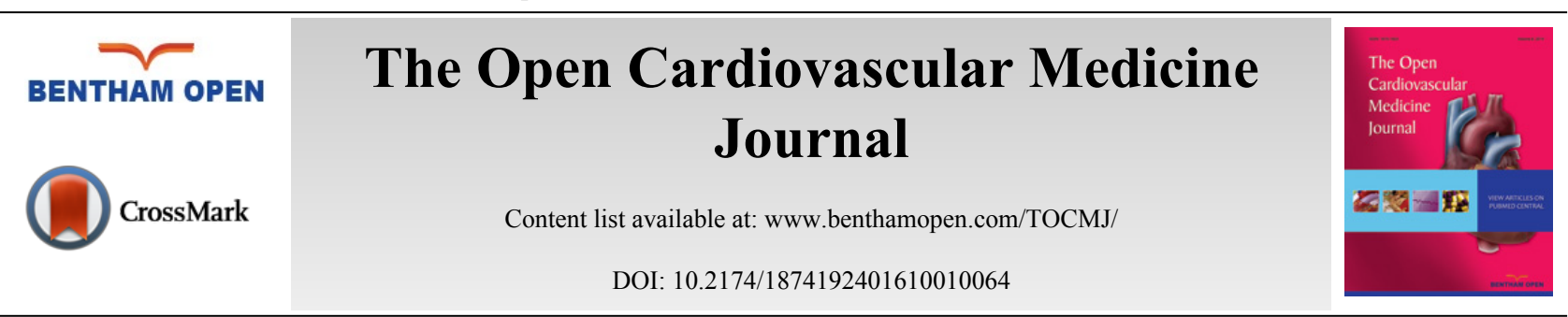

\title{
Is There an Association Between Carotid-Femoral Pulse Wave Velocity and Coronary Heart Disease in Patients with Coronary Artery Disease: A Pilot Study
}

\author{
Niki Katsiki ${ }^{1, *}$, Erietta Kollari ${ }^{1}$, Sotirios Dardas ${ }^{1}$, Petros Dardas ${ }^{2}$, Anna-Bettina Haidich ${ }^{3}$, Vasilios G. \\ Athyros $^{1}$ and Asterios Karagiannis ${ }^{1}$ \\ ${ }^{I}$ Department of Internal Medicine, Medical School, Aristotle University of Thessaloniki, Hip-pocration Hospital, \\ Thessaloniki, Greece \\ ${ }^{2}$ Department of Cardiology, Agios Loukas Hospital, Thessaloniki, Greece \\ ${ }^{3}$ Department of Hygiene and Epidemiology, Medical School, Aristotle University of Thessaloniki, Greece
}

Received: March 15, 2015

Revised: April 20, 2015

Accepted: April 25, 2015

\begin{abstract}
Arterial stiffness has been shown to predict cardiovascular morbidity and mortality. Carotid-femoral pulse wave velocity (cfPWV) is regarded the gold standard marker of arterial stiffness. In previous studies, cfPWV was associated with the presence of coronary heart disease (CHD). However, with regard to CHD severity as assessed by the Syntax Score, only brachial-ankle PWV was reported to correlate with Syntax Score; no data exist for cfPWV.

In this pilot study, we evaluated the possible associations between cfPWV, CHD and Syntax Score in 62 consecutive pa-tients (49 males; mean age: $64 \pm 12$ years) with chest pain undergoing scheduled coronary angiography. cfPWV was signifi-cantly higher in CHD patients than in non-CHD individuals $(10 v s .8 .4 \mathrm{~m} / \mathrm{s} ; p=0.003)$. No significant association was found between cfPWV and CHD severity as assessed by Syntax Score. A cut-off point of $12.3 \mathrm{~m} / \mathrm{s}$ was considered as diagnostic for abnormally increased cfPWV (specificity: 97\%; sensitivity: 12\%; positive likelihood ratio: 3.558). Further research is needed to establish the relationship between cfPWV and Syntax Score.
\end{abstract}

Keywords: Arterial stiffness, coronary angiography, coronary heart disease, pulse wave velocity, syntax score.

\section{INTRODUCTION}

Arterial stiffness represents an early manifestation of atherosclerosis characterized by adverse structural and functional alterations within the vessel wall. Markers of arterial stiffness include pulse pressure, augmentation index (AIx) and pulse-wave velocity (PWV), the latter being the most widely used index; carotid-femoral PWV (cfPWV) is regarded the gold standard for the measurement of arterial stiffness [1]. These markers have been related to increased risk for fatal and non-fatal cardiovascular disease (CVD) events [2].

Arterial stiffness has-been associated with several CVD risk factors such as increasing age, obesity, dyslipidemia, diabetes mellitus (DM), smoking and hypertension [3 - 9]. Other metabolic disorders related to increased CVD morbidity and/or mortality such as metabolic syndrome (MetS), non-alcoholic fatty liver disease (NAFLD), hyperuricemia, obstructive sleep apnea (OSAS) and chronic kidney disease (CKD), have also been linked to abnormal arterial stiffness [10 - 14].

Arterial stiffness (assessed by cfPWV) has been previously linked to the presence of coronary heart disease (CHD) [15 - 17]. Syntax score (SYNergy between percutaneous coronary intervention with TAXus and cardiac surgery) may

\footnotetext{
* Address correspondence to this author at the Department of Internal Medicine, Medical School, Aristotle University of Thessaloniki, Hip-pocration Hospital, 49 Konstantinoupoleos 49 Str, Thessaloniki, 54642 Greece; Tel: 00302310 992845; Fax: 00302310 835955; Email: astkar@med.auth.gr
} 
be used to evaluate CHD complexity, severity and clinical prognosis in various patient subgroups undergoing percutaneous coronary interventions (PCIs) [18]. Only 2 studies evaluated the association between Syntax Score (as a marker of CHD severity) and brachial-ankle PWV (baPWV) [19, 20] reporting significant correlations between the 2 variables. However, no data exist with regard to cfPWV and Syntax score.

We evaluated the possible associations between cfPWV, CHD and Syntax Score in patients with stable angina undergoing scheduled coronary angiography.

\section{PATIENTS AND METHODS}

A total of 62 consecutive patients with chest pain (49 males; mean age:64, SD: 12 years) undergoing a scheduled coronary angiography at the Cardiology Department of Agios Loukas Hospital, Thessaloniki, Greece, were included in the present pilot study. The study was approved by the local ethics committee and informed consent was obtained from all participants.

Prior to coronary angiography, a detailed individual and family history was recorded and physical examinations including weight, height, heart rate and blood pressure measurements, as well as laboratory analyses serum creatinine, total cholesterol, triglycerides, high density lipoprotein cholesterol (HDL-C) and low density lipoprotein cholesterol (LDL-C) were performed in all patients. Furthermore, arterial stiffness was assessed by the measurement of cfPWV with an automated applanation tonometry device i.e. SpygmoCor (AtCor Medical, Sydney, Australia). AIx75 (AIxadjusted to a standard heart rate of $75 \mathrm{bpm}$ ) was also recorded. Glomerular filtration rate (GFR) was calculated based on the Modification of Diet in Renal Disease (MDRD) formula $186.3 \times($ serum creatinine in $\mathrm{mg} / \mathrm{dl}-1.154) \times(\mathrm{age}$ in years- 0.203$) \times 1.212$ (if black) $\times 0.742$ (if female).

Syntax score is calculated based on angiographical findings; an online calculator was used to generate Syntax score (http://www.syntaxscore.com).

Exclusion criteria were acute or chronic infections, non-cardiac chest pain, cancer, systematic diseases, major surgical procedures during the last 3 months, history of acute myocardial infarction, cardiac arrhythmias, cardiomyopathies, carotid sinus syndrome, previous percutaneous coronary intervention (PCI), severe carotid stenosis and peripheral arterial disease.

Following coronary angiography, the study population was divided into 2 groups: patients with angiographically proven CHD i.e. those with $>50 \%$ stenosis $(n=33)$ and non-CHD individuals $(n=29)$.

\section{Statistical Analysis}

Statistical analyses were performed using the SPSS version 23.0 software package (SPSS Inc., Chicago, USA). Normally distributed continuous variables were expressed as mean and standard deviation (SD), whereas non-normally distributed ones were expressed as median and interquartile range(IQR); categorical values were presented as frequencies with percentages. Differences between the 2 study groups as well as other subgroups (i.e. diabetic, dyslipidemic and hypertensive patients) were evaluated by Student's t- test, or the Mann Whitney U test, whenever more appropriate. The association between categorical variables was assessed with the chi-square test or the Fisher's exact test whenever more appropriate. Correlations between continuous variables were examined by Pearson $r$ or Spearman Rho correlation coefficients. Logistic regression analysis was performed and odds ratios were presented with the corresponding 95\% confidence intervals (OR, 95\% CI). A multivariable model was built with backward elimination based on likelihood ratio criteria, where univariate predictors with P-values lower than 0.05 were considered for inclusion. The model fit was evaluated with the Hosmer-Lemeshow test. Discrimination performance of the multivariable model was evaluated with the C-index. Furthermore, receiver operating characteristic (ROC) curves analysis was used to determine the cut-off point for the diagnosis of abnormally increased cfPWV. A 2-tailed $p<0.05$ was considered as significant.

\section{RESULTS}

Table 1 summarizes the differences between CHD and non-CHD patients in all studied variables. There were significantly more males in the CHD group compared with the non-CHD group $(91 v s .65 \% ; p=0.014)$ as well as patients with a history of dyslipidemia (91 vs 69\%, respectively; $\mathrm{p}=0.029)$. Triglycerides levels were also greater in the CHD group compared with the non-CHD group [137 $(94-=0.044]$. Similarly, cfPWV was significantly higher in CHD patients than in non-CHD individuals (10 vs $8.4 \mathrm{~m} / \mathrm{s} ; p=0.003$ ), whereas AIx 75 did not differ between the 2 groups. No 
significant association was observed between cfPWV and CHD severity as assessed by Syntax Score $(\mathrm{r}=0.038 ; p=$ 0.833). Furthermore, CHD patients were more likely to receive a statin than non-CHD individuals $(66.7 v s 41.4 \% ; p=$ $0.046)$.

In multivariable analysis, only cfPWV remained significantly different between the 2 groups (Table 2). The Hosmer and Lemeshow test indicated a good fit of the data $(p=0.585)$ and the discriminative performance of the model was good $(\mathrm{C}$-index $=0.81)$ The model fit was assessed with the Hosmer and Lemeshow test $(p=0.585)$ with a $\mathrm{C}$-index of 0.81 , indicating a good power and fit of the model.

Table 1. Differences between patient groups in all studied variables.

\begin{tabular}{|c|c|c|c|}
\hline & $\begin{array}{c}\text { CHD } \\
\mathrm{n}=\mathbf{3 3} \\
\end{array}$ & $\begin{array}{c}\begin{array}{c}\text { Non CHD } \\
\mathrm{n}=\mathbf{2 9}\end{array} \\
\end{array}$ & $p$ \\
\hline Gender, male, $n(\%)$ & $30(91)$ & $19(65)$ & 0.014 \\
\hline Mean height,meters (SD) & $1.72(0.8)$ & $1.70(0.8)$ & 0.539 \\
\hline Mean weight in kg (SD) & $85.1(14.8)$ & $91.7(18.1)$ & 0.123 \\
\hline Mean age in years (SD) & $64(12)$ & $63(11)$ & 0.569 \\
\hline History of Hypertension, $n(\%)$ & $22(66$ & $23(79$ & 0.265 \\
\hline Current Smokers, n (\%) & $13(39)$ & $6(20)$ & 0.111 \\
\hline History of Diabetes, n (\%) & $10(30$ & $4(14)$ & 0.121 \\
\hline History of Dyslipidemia, $\mathrm{n}(\%)$ & $30(91)$ & $20(69)$ & $\mathbf{0 . 0 2 9}$ \\
\hline Family history of CHD, n (\%) & $15(45)$ & $7(24)$ & 0.080 \\
\hline Median GFR $\mathrm{mL} / \mathrm{min} / 1.73 \mathrm{~m} 2$ (IQR) & $87.5(67-94)$ & $81.2(68-96)$ & 0.849 \\
\hline Median Total cholesterol, mg/dL (IQR) & $162(147-209)$ & $180(158-203)$ & 0.476 \\
\hline Median LDL-C, mg/dL (IQR) & $101(81-145)$ & $110(93-135)$ & 0.631 \\
\hline Median HDL-C, mg/dL (IQR) & $44(36-53)$ & $52(39-57)$ & 0.156 \\
\hline Median Triglycerides, mg/dL (IQR) & $137(94-179)$ & $106(87-142)$ & 0.044 \\
\hline Mean Heart rate, bpm (SD) & $68(10)$ & $66(9)$ & 0.598 \\
\hline Median SBP, mmHg (IQR) & $120(110-140)$ & $125(110-135)$ & 0.679 \\
\hline Median DBP, mmHg (IQR) & $80(70-83)$ & $80(70-90)$ & 0.878 \\
\hline Mean AIx75 (SD) & $21.1(10.6)$ & $21.8(10.7)$ & 0.796 \\
\hline Median cfPWV, m/s (SD) & $10(9-11)$ & $8.4(7.6-9.8)$ & 0.003 \\
\hline LVH, n (\%) & $17(51)$ & $9(31)$ & 0.103 \\
\hline Nitrates, n (\%) & $9(27.3)$ & $3(10.3)$ & 0.092 \\
\hline$\beta$-Blockers, $n(\%)$ & $21(63.6)$ & $16(55.2)$ & 0.498 \\
\hline ACE inhibitors, $\mathrm{n}(\%)$ & $4(12.1)$ & $5(17.2)$ & 0.568 \\
\hline Angiotensin receptor blockers, n (\%) & $18(54.5)$ & $10(34.5)$ & 0.113 \\
\hline Calcium channel blockers, n (\%) & $12(36.4)$ & $7(24.1)$ & 0.297 \\
\hline Diuretics, n (\%) & $16(48.5)$ & $8(27.6)$ & 0.092 \\
\hline Statins, n (\%) & $22(66.7)$ & $12(41.4)$ & 0.046 \\
\hline Aldactone, n (\%) & $2(6.9)$ & $1(3)$ & 0.595 \\
\hline
\end{tabular}

CHD: coronary heart disease; GFR: glomerular filtration rate; LDL-C: low density lipoprotein cholesterol; HDL-C: high density lipoprotein cholesterol; SBP: systolic blood pressure; DBP: diastolic blood pressure; AIx75: augmentation index adjusted to a standard heart rate of 75bpm; cfPWV: carotid-femoral pulse wave velocity; LVH: left ventricular hypertrophy; ACE:angiotensinconvertingenzyme; SD:Standard deviation; IQR: Interquartile range

\section{DISCUSSION}

We evaluated the associations between cfPWV, CHD and Syntax Score in patients with chest pain undergoing a scheduled coronary angiography. Significantly more males and patients with a history of dyslipidemia were observed in the CHD group than the non-CHD group, as expected. CHD patients were more likely to receive a statin and had higher triglycerides levels compared with non-CHD individuals.

With regard to arterial stiffness, cfPWV was independently associated with CHD prevalence (but not AIx75). These findings are in agreement with previous studies [15 - 17]. However, a link between AIx75 and a composite endpoint of myocardial infarction, stroke, cardiac, cerebrovascular, and peripheral revascularization as well as total mortality has been reported in Austrian males $(n=520)$ undergoing coronary angiography [21].

We found no significant association between cfPWV and CHD severity as assessed by Syntax Score. To our knowledge, this is the first study to estimate the relationship between cfPWV and Syntax Score. In contrast, Syntax Score has been previously related to baPWV [19, 20].

In this study, the value of $12.3 \mathrm{~m} / \mathrm{s}$ was characterized as the cut-off point for the diagnosis of abnormally increased cfPWV in this patient population (specificity: 97\%; sensitivity: 12\%). This is similar to the cut-off point for cfPWV (i.e. 
$12 \mathrm{~m} / \mathrm{s}$ ) suggested by the Task Force for the Management of Arterial Hypertension of the European Society of Hypertension (ESH) and the European Society of Cardiology (ESC) in 2007 to estimate subclinical atherosclerosis [22]. However, in the more recent $2013 \mathrm{ESH} / \mathrm{ESC}$ guidelines for the management of arterial hypertension, the cfPWV cut-off point for asymptomatic organ damage was $10 \mathrm{~m} / \mathrm{s}$ [23].

Table 2. Factors associated with CHD.

\begin{tabular}{|c|c|c|c|}
\hline & OR & 95\% CI & p \\
\hline Gender & 4.9 & 0.9 & -26.5 \\
\hline History of dyslipidemia & 0.45 & $0.09-2.4$ & 0.065 \\
\hline cfPWV & 1.38 & $1.04-1.85$ & 0.347 \\
\hline
\end{tabular}

OR: odds ratio; CI: confidence intervals; CHD: coronary heart disease; cfPWV:carotid-femoral pulse wave velocity

Antihypertensive drugs have been consistently shown to improve arterial stiffness [24 - 26]. Similar beneficial effects may be exerted by statins [27]. Of note, antihypertensive therapy did not differ between groups in the present study, whereas patients with CHD were more likely to use statin with a marginal $p$ value $(p=0.046)$.

Our study has limitations including the small sample size, recruitment of patients from one clinical center and its cross-sectional design. However, this is a pilot study and to our knowledge the first to evaluate the association between cfPWV and Syntax Score.

\section{CONCLUSION}

cfPWV was independently associated with CHD prevalence in patients with chest pain undergoing scheduled coronary angiography. Patients with DM or dyslipidemia had higher cfPWV values than individuals without these metabolic disorders. However, no significant association was observed between cfPWV and CHD severity as assessed by SyntaxScore.A cut-off point of $12.3 \mathrm{~m} / \mathrm{s}$ for the diagnosis of abnormally increased cfPWVmay be used in this patient population. Further research is needed to establish the relationship between cfPWV and Syntax Score.

\section{CONFLICT OF INTEREST}

This original study was conducted independently. The authors did not receive financial or professional help with the preparation of the manuscript. The authors have given talks, attended conferences and participated in advisory boards and trials sponsored by various pharmaceutical companies.

\section{ACKNOWLEDGEMENTS}

Declared none.

\section{REFERENCES}

[1] Laurent S, Boutouyrie P. Arterial stiffness: a new surrogate end point for cardiovascular disease? J Nephrol 2007; 20(Suppl. 12): S45-50. [PMID: 18050143]

[2] Liao J, Farmer J. Arterial stiffness as a risk factor for coronary artery disease. Curr Atheroscler Rep 2014; $16(2): 387$. [http://dx.doi.org/10.1007/s11883-013-0387-8] [PMID: 24402301]

[3] Cheitlin MD. Cardiovascular physiology-changes with aging. Am J Geriatr Cardiol 2003; 12(1): 9-13. [http://dx.doi.org/10.1111/j.1076-7460.2003.01751.x] [PMID: 12502909]

[4] Wilkinson I, Cockcroft JR. Cholesterol, lipids and arterial stiffness. Adv Cardiol 2007; 44: 261-77. [http://dx.doi.org/10.1159/000096747] [PMID: 17075215]

[5] Doonan RJ, Hausvater A, Scallan C, Mikhailidis DP, Pilote L, Daskalopoulou SS. The effect of smoking on arterial stiffness. Hypertens Res 2010; 33(5): 398-410.

[http://dx.doi.org/10.1038/hr.2010.25] [PMID: 20379189]

[6] Seifalian AM, Filippatos TD, Joshi J, Mikhailidis DP. Obesity and arterial compliance alterations. Curr Vasc Pharmacol 2010; 8(2): 155-68. [http://dx.doi.org/10.2174/157016110790886956] [PMID: 20180777]

[7] Sudano I, Roas S, Noll G. Vascular abnormalities in essential hypertension. Curr Pharm Des 2011; 17(28): $3039-44$. [http://dx.doi.org/10.2174/138161211798157766] [PMID: 21861833]

[8] Athyros VG, Katsiki N, Doumas M, Karagiannis A, Mikhailidis DP. Effect of tobacco smoking and smoking cessation on plasma lipoproteins and associated major cardiovascular risk factors: a narrative review. Curr Med Res Opin 2013; 29(10): 1263-74. [http://dx.doi.org/10.1185/03007995.2013.827566] [PMID: 23879722]

[9] Christen AI, Armentano RL, Miranda A, et al. Arterial wall structure and dynamics in type 2 diabetes mellitus methodological aspects and pathophysiological findings. Curr Diabetes Rev 2010; 6(6): 367-77. 
[http://dx.doi.org/10.2174/157339910793499146] [PMID: 20879975]

[10] Katsiki N, Athyros VG, Karagiannis A, Mikhailidis DP. Characteristics other than the diagnostic criteria associated with metabolic syndrome: an overview. Curr Vasc Pharmacol 2014; 12(4): 627-41. [http://dx.doi.org/10.2174/15701611113119990131] [PMID: 23627982]

[11] Oni ET, Agatston AS, Blaha MJ, et al. A systematic review: burden and severity of subclinical cardiovascular disease among those with nonalcoholic fatty liver; should we care? Atherosclerosis 2013; 230(2): 258-67. [http://dx.doi.org/10.1016/j.atherosclerosis.2013.07.052] [PMID: 24075754]

[12] Bae JS, Shin DH, Park PS, et al. The impact of serum uric acid level on arterial stiffness and carotid atherosclerosis: the Korean Multi-Rural Communities Cohort study. Atherosclerosis 2013; 231(1): 145-51.

[http://dx.doi.org/10.1016/j.atherosclerosis.2013.08.017] [PMID: 24125426]

[13] Phillips CL, Butlin M, Wong KK, Avolio AP. Is obstructive sleep apnoea causally related to arterial stiffness? A critical review of the experimental evidence. Sleep Med Rev 2013; 17(1): 7-18. [http://dx.doi.org/10.1016/j.smrv.2012.03.002] [PMID: 22658640]

[14] Gungor O, Kircelli F, Voroneanu L, Covic A, Ok E. Hormones and arterial stiffness in patients with chronic kidney disease. J Atheroscler Thromb 2013; 20(9): 698-707. [http://dx.doi.org/10.5551/jat.18580] [PMID: 23911970]

[15] Vakalis K, Bechlioulis A, Naka KK, et al. Role of 9p21 and 2q36 variants and arterial stiffness in the prediction of coronary artery disease. Eur J Clin Invest 2014; 44(8): 784-94.

[http://dx.doi.org/10.1111/eci.12295] [PMID: 24942486]

[16] Mansour AS, Yannoutsos A, Majahalme N, et al. Aortic stiffness and cardiovascular risk in type 2 diabetes. J Hypertens 2013; 31(8): 1584-92. [http://dx.doi.org/10.1097/HJH.0b013e3283613074] [PMID: 23822928]

[17] Bechlioulis A, Vakalis K, Naka KK, et al. Increased aortic pulse wave velocity is associated with the presence of angiographic coronary artery disease in overweight and obese patients. Am J Hypertens 2013; 26(2): 265-70. [http://dx.doi.org/10.1093/ajh/hps039] [PMID: 23382412]

[18] Chakrabarti AK, Gibson CM. The SYNTAX score: usefulness, limitations, and future directions. J Invasive Cardiol 2011; 23 (12): 511-2. [PMID: 22147398]

[19] Xiong Z, Zhu C, Zheng Z, et al. Relationship between arterial stiffness assessed by brachial-ankle pulse wave velocity and coronary artery disease severity assessed by the SYNTAX score. J Atheroscler Thromb 2012; 19(11): 970-6. [http://dx.doi.org/10.5551/jat.13326] [PMID: 22785141]

[20] Chung CM, Yang TY, Lin YS, et al. Relation of arterial stiffness assessed by brachial-ankle pulse wave velocity to complexity of coronary artery disease. Am J Med Sci 2014; 348(4): 294-9. [http://dx.doi.org/10.1097/MAJ.0000000000000285] [PMID: 24845468]

[21] Weber T, O’Rourke MF, Lassnig E, et al. Pulse waveform characteristics predict cardiovascular events and mortality in patients undergoing coronary angiography. J Hypertens 2010; 28(4): 797-805. [http://dx.doi.org/10.1097/HJH.0b013e328336c8e9] [PMID: 20164805]

[22] Mancia G, De Backer G, Dominiczak A, et al. Guidelines for the management of arterial hypertension. J Hypertens 2007; $25(6)$ : $1105-87$. [http://dx.doi.org/10.1097/HJH.0b013e3281fc975a] [PMID: 17563527]

[23] Mancia G, Fagard R, Narkiewicz K, et al. 2013 ESH/ESC Guidelines for the management of arterial hypertension: the Task Force for the management of arterial hypertension of the European Society of Hypertension (ESH) and of the European Society of Cardiology (ESC). J Hypertens 2013; 31(7): 1281-357. [http://dx.doi.org/10.1097/01.hjh.0000431740.32696.cc] [PMID: 23817082]

[24] Ong KT, Delerme S, Pannier B, et al. Aortic stiffness is reduced beyond blood pressure lowering by short-term and long-term antihypertensive treatment: a meta-analysis of individual data in 294 patients. J Hypertens 2011; 29(6): 1034-42. [http://dx.doi.org/10.1097/HJH.0b013e328346a583] [PMID: 21519280]

[25] Koumaras C, Tzimou M, Stavrinou E, et al. Role of antihypertensive drugs in arterial 'de-stiffening' and central pulsatile hemodynamics. Am J Cardiovasc Drugs 2012; 12(3): 143-56. [http://dx.doi.org/10.2165/11599040-000000000-00000] [PMID: 22540323]

[26] Koumaras C, Tziomalos K, Stavrinou E, et al. Effects of renin-angiotensin-aldosterone system inhibitors and beta-blockers on markers of arterial stiffness. J Am Soc Hypertens 2014; 8(2): 74-82. [http://dx.doi.org/10.1016/j.jash.2013.09.001] [PMID: 24139833]

[27] Papademetriou V, Katsiki N, Doumas M, Faselis C. Halting arterial aging in patients with cardiovascular disease: hypolipidemic and antihypertensive therapy. Curr Pharm Des 2014; 20(40): 6339-49. [http://dx.doi.org/10.2174/1381612820666140620162157] [PMID: 24953392] 